\title{
BMJ
}

\section{Pre-eclampsia, soluble fms-like tyrosine kinase 1, and the risk of reduced thyroid function: nested case-control and population based study}

\author{
Richard I Levine, senior investigator, ${ }^{1}$ Lars I Vatten, professor, ${ }^{2}$ Gary L Horowitz, associate professor, ${ }^{3}$ Cong \\ Qian, statistician, ${ }^{4}$ Pal R Romundstad, associate professor, ${ }^{2}$ Kai F Yu, senior statistician, ${ }^{1}$ Anthony N \\ Hollenberg, associate professor, ${ }^{5}$ Alf I Hellevik, medical student, ${ }^{2}$ Bjorn 0 Asvold, postdoctoral fellow, ${ }^{2} \mathrm{~S}$ \\ Ananth Karumanchi, associate professor ${ }^{5,6,7}$
}

${ }^{1}$ Department of Health and Human Services, Eunice Kennedy Shriver National Institute of Child Health and Human Development, Division of Epidemiology, Statistics, and Prevention Research, Bethesda, MD 20892, USA

${ }^{2}$ Department of Public Health, Faculty of Medicine, Norwegian University of Science and Technology, Trondheim, Norway

${ }^{3}$ Department of Pathology, Beth Israel Deaconess Medical Center and Harvard Medical School, Boston, MA, USA

${ }^{4}$ Glotech, Rockville, MD, USA

${ }^{5}$ Department of Medicine, Beth Israel Deaconess Medical Center and Harvard Medical School, Boston, MA, USA

${ }^{6}$ Department of Obstetrics and Gynecology, Beth Israel Deaconess Medical Center and Harvard Medical School, Boston, MA, USA

${ }^{7}$ Howard Hughes Medical Institute, Boston, MA, USA

Correspondence to: RJ Levine LevineRJ@mail.nih.gov or S A Karumanchi

sananth@bidmc.harvard.edu

Cite this as: $B M J$ J 2009;339:b4336 doi:10.1136/bmj.b4336

\section{ABSTRACT}

Objective To determine if pre-eclampsia is associated with reduced thyroid function during and after pregnancy. Design Nested case-control study during pregnancy and population based follow-up study after pregnancy. Setting Calcium for Pre-eclampsia Prevention trial of healthy pregnant nulliparous women in the United States during 1992-5, and a Norwegian population based study (Nord-Trondelag Health Study or HUNT-2) during 1995-7 with linkage to the medical birth registry of Norway.

Participants All 141 women (cases) in the Calcium for Preeclampsia Prevention trial with serum measurements before 21 weeks' gestation (baseline) and after onset of pre-eclampsia (before delivery), 141 normotensive controls with serum measurements at similar gestational ages, and 7121 women in the Nord-Trondelag Health Study whose first birth had occurred in 1967 or later and in whom serum levels of thyroid stimulating hormone had been subsequently measured.

Main outcome measures Thyroid function tests and human chorionic gonadotrophin and soluble fms-like tyrosine kinase 1 concentrations in the Calcium for Preeclampsia Prevention cohort and odds ratios for levels of thyroid stimulating hormone above the reference range, according to pre-eclampsia status in singleton pregnancies before the Nord-Trondelag Health Study. Results In predelivery specimens of the Calcium for Preeclampsia Prevention cohort after the onset of preeclampsia, thyroid stimulating hormone levels increased 2.42 times above baseline compared with a 1.48 times increase in controls. The ratio of the predelivery to baseline ratio of cases to that of the controls was 1.64 (95\% confidence interval 1.29 to 2.08 ). Free triiodothyronine decreased more in the women with preeclampsia than in the controls (case ratio to control ratio $0.96,95 \%$ confidence interval 0.92 to 0.99 ). The predelivery specimens but not baseline samples from women with pre-eclampsia were significantly more likely than those from controls to have concentrations of thyroid stimulating hormone above the reference range (adjusted odds ratio $2.2,95 \%$ confidence interval 1.1 to 4.4 ). Both in women who developed pre-eclampsia and in normotensive controls the increase in thyroid stimulating hormone concentration between baseline and predelivery specimens was strongly associated with increasing quarters of predelivery soluble fms-like tyrosine kinase 1 (P for trend 0.002 and $<0.001$, respectively). In the NordTrondelag Health Study, women with a history of preeclampsia in their first pregnancy were more likely than other women (adjusted odds ratio 1.7, 95\% confidence interval 1.1 to 2.5 ) to have concentrations of thyroid stimulating hormone above the reference range ( $>3.5$ $\mathrm{mIU} / \mathrm{l})$. In particular, they were more likely to have high concentrations of thyroid stimulating hormone without thyroid peroxidase antibodies (adjusted odds ratio 2.6, $95 \%$ confidence interval 1.3 to 5.0 ), suggesting hypothyroid function in the absence of an autoimmune process. This association was especially strong $(5.8,1.3$ to 25.5) if pre-eclampsia had occurred in both the first and the second pregnancies.

Conclusion Increased serum concentration of soluble fms-like tyrosine kinase 1 during pre-eclampsia is associated with subclinical hypothyroidism during pregnancy. Pre-eclampsia may also predispose to reduced thyroid function in later years.

\section{INTRODUCTION}

Pre-eclampsia, a pregnancy specific syndrome characterised by new onset hypertension and proteinuria, causes substantial morbidity and mortality in mothers and infants. ${ }^{12}$ Women with a history of pre-eclampsia have an increased risk of dyslipidaemia, hypertension, and cardiovascular and renal disease..$^{3-6}$

Although the cause of pre-eclampsia is still unclear, studies in both humans and animals suggest that excess circulating antiangiogenic factors such as soluble fmslike tyrosine kinase 1 (sFlt-1 or sVEGFR1) may be responsible for the clinical phenotype of preeclampsia. ${ }^{7-9}$ Blood concentrations of soluble fms-like tyrosine kinase 1 increase during the last two months of normal pregnancy and increase to much greater levels in women with pre-eclampsia. Soluble fms-like 
tyrosine kinase 1 acts by inhibiting vascular endothelial growth factor and placental growth factor signalling. Indeed, the use of vascular endothelial growth factor inhibitors for the treatment of cancer related angiogenesis has been associated with hypertension, proteinuria, glomerular endothelial damage, increased concentrations of circulating liver enzymes, cerebral oedema, and reversible posterior leucoencephalopathy - a constellation of conditions resembling those found in women with pre-eclampsia or eclampsia. ${ }^{1011}$

More recently, patients with cancer who had received prolonged therapy with vascular endothelial growth factor inhibitors were found to be at greater risk of hypothyroidism. ${ }^{12-14}$ Furthermore, studies in mice using vascular endothelial growth factor inhibitors such as soluble fms-like tyrosine kinase 1 have shown substantial thyroid capillary regression and increased concentrations of thyroid stimulating hormone. ${ }^{115}$ We therefore hypothesised that the excess soluble fms-like tyrosine kinase 1 accompanying pre-eclampsia might be associated with reduced thyroid function during pregnancy and that women who have experienced pre-eclampsia would have an increased risk of hypothyroid function later in life.

To compare thyroid function in women who developed pre-eclampsia with those who remained normotensive during pregnancy, we carried out a nested casecontrol study within the Calcium for Pre-eclampsia Prevention (CPEP) trial cohort. We hypothesised that women with pre-eclampsia would experience a greater increase in thyroid stimulating hormone concentration during pregnancy than normotensive controls and that the extent of the increase would correlate with the magnitude of the soluble fms-like tyrosine kinase 1 concentration during pre-eclampsia. In addition, we used a Norwegian population based cohort study (the NordTrondelag Health Study or HUNT-2) to test whether pre-eclampsia in a previous pregnancy is associated with risk of reduced thyroid function in later life.

\section{Calcium for Pre-eclampsia Prevention trial}

\section{Participants and specimens}

The Calcium for Pre-eclampsia Prevention trial was a randomised, double blind clinical trial carried out during 1992-5 in healthy nulliparous women with singleton pregnancies to evaluate the effects of daily supplementation with calcium or placebo on the incidence and severity of pre-eclampsia. ${ }^{16}{ }^{17}$ Calcium supplementation did not reduce the incidence or severity of pre-eclampsia or delay its onset.

Of the 4589 women enrolled in the trial we excluded 300 with incomplete information on outcomes or whose pregnancy ended before 21 weeks. Of 326 women who developed pre-eclampsia, 141 had at least one serum specimen collected before 21 weeks' gestation (baseline specimen) and one collected after the onset of pre-eclampsia (predelivery specimen). If a woman had more than one specimen collected within each of these intervals, we selected the earliest before 21 weeks and the latest after the onset of pre-eclampsia.
Soluble fms-like tyrosine kinase 1 had previously been analysed in all serum specimens of a random sample of 2200 women and in all women with pre-eclampsia. ${ }^{18}$ After excluding women with pre-eclampsia, gestational hypertension, or gestational proteinuria, 1649 women remained who had been normotensive and without proteinuria during their pregnancies (controls). Each case of pre-eclampsia was matched to the control with two serum specimens that were closest in gestation to the two case specimens. Two case specimens-one baseline, one predelivery-were not located in the specimen repository.

Women with active dysfunction of the thyroid that required drugs were excluded from the Calcium for Pre-eclampsia Prevention trial except those with hypothyroidism who were stable while receiving thyroid replacement therapy. A check of drugs reported by the participants of this study showed that only one woman (who later developed pre-eclampsia) had received thyroxine. This woman had subclinical hypothyroidism at baseline, but concentrations of thyroid stimulating hormone and free thyroxine in her predelivery specimen were within clinical reference ranges.

Pre-eclampsia was defined as hypertension - that is, a diastolic blood pressure of at least $90 \mathrm{~mm} \mathrm{Hg}$ on two occasions four to 168 hours apart-and proteinuria, characterised as one of the following: urine dipstick results of at least $+(30 \mathrm{mg} / \mathrm{dl})$ on two occasions four to 168 hours apart; a protein to creatinine ratio of at least 0.35 ; urine dipstick results of at least ++ $(100 \mathrm{mg} / \mathrm{dl})$, or a 24 hour urine specimen containing at least $300 \mathrm{mg}$ of protein. Detailed definitions have been published previously. ${ }^{16} 17$

\section{Procedures}

We randomly ordered archived serum specimens, which had been stored at $-70^{\circ} \mathrm{C}$, for analysis. Assays were carried out by staff who were unaware of the outcome of the pregnancy. Enzyme linked immunosorbent assays for human soluble fms-like tyrosine kinase 1 had previously been done in duplicate by R\&D Systems Analytical Testing Services (Minneapolis, MN, USA).$^{18}$ Thyroid function tests (thyroid stimulating hormone, free thyroxine, free triiodothyronine, thyroid peroxidase antibodies) were carried out and human chorionic gonadotrophin measured using a Roche Diagnostics Modular Analytics E170 analyzer (Roche Diagnostics, Indianapolis, IN, USA). Human chorionic gonadotrophin was measured because it is known to stimulate the thyroid gland and to decrease concentrations of thyroid stimulating hormone. ${ }^{19}$ The Roche human chorionic gonadotrophin assay shows no cross reactivity with thyroid stimulating hormone. Coefficients of variation in normal serum in mid-pregnancy were less than 5\%. Reference ranges for thyroid stimulating hormone $(\mathrm{mIU} / \mathrm{l})$ provided by the manufacturer were $0.33-4.60,0.35-4.10$, and $0.21-3.15$ in sera from the first, second, and third trimester, respectively. The upper limits of the reference ranges for thyroid peroxidase antibodies $(\mathrm{IU} / \mathrm{ml})$ provided by the 
Table 1|Characteristics of women with pre-eclampsia and controls at enrolment in Calcium for Pre-eclampsia Prevention trial and characteristics of their infants and specimens*. Values are numbers (percentages) unless stated otherwise

\begin{tabular}{|c|c|c|c|}
\hline Characteristics & $\begin{array}{l}\text { Cases } \\
(n=141)\end{array}$ & $\begin{array}{l}\text { Controls } \\
(n=141)\end{array}$ & $P$ value \\
\hline \multicolumn{4}{|l|}{ Women } \\
\hline Mean (SD) age (years) & $20.9(4.0)$ & $20.1(3.8)$ & 0.09 \\
\hline Mean (SD) height (cm) & $161(7)$ & $162(8)$ & 0.08 \\
\hline Mean (SD) weight (kg) & $71.9(19.0)$ & $65.3(15.4)$ & 0.002 \\
\hline Mean (SD) body mass index $\dagger$ & 27.7 (6.9) & $24.7(5.2)$ & $<0.001$ \\
\hline Mean (SD) systolic blood pressure (mm Hg) & $111(8)$ & $107(9)$ & $<0.001$ \\
\hline Mean (SD) diastolic blood pressure (mm Hg) & $63(8)$ & $60(8)$ & 0.003 \\
\hline Mean (SD) gestational age at enrolment (weeks) & $17.4(2.6)$ & $17.5(2.5)$ & 0.94 \\
\hline Current smoker & $11(8)$ & $23(16)$ & 0.03 \\
\hline Calcium treatment & $62(44)$ & $64(45)$ & 0.81 \\
\hline Private health insurance & $8(6)$ & $12(9)$ & 0.35 \\
\hline Ever married & $33(23)$ & 27 (19) & 0.38 \\
\hline \multicolumn{4}{|l|}{ Race or ethnic groupł: } \\
\hline White, non-Hispanic & $35(24.8)$ & $56(39.7)$ & \multirow{4}{*}{0.02} \\
\hline White, Hispanic & $19(13.5)$ & $24(17.0)$ & \\
\hline Black & $81(57.5)$ & $58(41.1)$ & \\
\hline Other or unknown & $6(4.3)$ & $3(2.1)$ & \\
\hline \multicolumn{4}{|l|}{ Infants } \\
\hline Mean (SD) birth weight (g) & $2964(816)$ & $3290(410)$ & $<0.001$ \\
\hline Delivery at <37 weeks & $40(28.4)$ & $3(2.1)$ & $<0.001$ \\
\hline Small for gestational age ( $(10$ th centile)§ & $25(17.7)$ & $8(5.7)$ & 0.002 \\
\hline \multicolumn{4}{|l|}{ Specimens } \\
\hline Mean (range) gestational age of baseline specimen (days) & $111(42-146)$ & $112(49-147)$ & 0.45 \\
\hline Mean (range) gestational age of predelivery specimen (days) & $265(156-296)$ & $260(166-292)$ & 0.04 \\
\hline
\end{tabular}

manufacturer were 119,91 , and 171 in sera from the first, second, and third trimester, respectively. ${ }^{20}$ Women with values above the reference range were considered to have tested positive for thyroid peroxidase antibodies.

\section{Statistical analysis}

We compared categorical variables using the $\chi^{2}$ test and continuous variables using $t$ tests. The geometric means of thyroid stimulating hormone, free thyroxine, free triiodothyronine, and human chorionic gonadotrophin and their standard deviations are reported for baseline and predelivery specimens for both cases and controls. Comparisons of cases and controls are presented as two tailed P values. Statistical comparisons of specimens from cases and controls were carried out using linear models, adjusting for age, body mass index, race or ethnicity (black people $v$ others), smoking status (current smoker or quit during pregnancy $v$ never smoker or quit before pregnancy), human chorionic gonadotrophin concentration, and presence of thyroid peroxidase antibodies above the reference range. The levels of change from baseline to predelivery are presented as ratios. Geometric means of the ratios and their standard deviations are given. We present the comparison statistic between case and control, which is the ratio of their geometric means, with its $95 \%$ confidence interval.

Results

\section{Characteristics of the women}

Of the 141 women with pre-eclampsia, $63(42 \%)$ had severe pre-eclampsia and in $47(33 \%)$ pre-eclampsia began before 37 weeks' gestation (table 1). Compared with the controls, women with pre-eclampsia had a greater body mass index and higher blood pressure at the time of enrolment in the Calcium for Pre-eclampsia Prevention trial. Moreover, a larger proportion of their current pregnancies had been complicated by preterm delivery or had resulted in the delivery of small for gestational age infants (table 1). The difference in gestational age between baseline and predelivery specimens in cases and controls did not differ significantly (154 v 148 days, $\mathrm{P}=0.08$ ).

With one exception the characteristics of the 141 women with pre-eclampsia and their infants from the Calcium for Pre-eclampsia Prevention trial included in this study (table 1) did not differ significantly from those of the 185 women with pre-eclampsia and their infants who had not been included owing to lack of specimens: infants of the women who had been included were more often delivered preterm $(28 \% \mathrm{v}$ $16 \%, \mathrm{P}=0.006)$.

\section{Thyroid function tests and pre-eclampsia}

The mean values of thyroid function tests at baseline were not significantly different (table 2). However, after the onset of pre-eclampsia, concentrations of thyroid stimulating hormone and human chorionic gonadotrophin were higher in cases than in controls. The magnitude of the increase in thyroid stimulating hormone concentration from baseline to predelivery (predelivery to baseline ratio) was greater in cases than in controls (table 2); case ratio to control ratio 1.64 (95\% confidence interval 1.29 to 2.08). Compared with baseline specimens, levels of free triiodothyronine were lower in predelivery specimens, and the magnitude of the decrease was significantly greater in cases than in controls (predelivery to baseline ratio $0.85 v 0.89$, case ratio to control ratio $0.96,0.92$ to 0.99 ). Although free thyroxine concentrations were also lower in predelivery specimens than in baseline specimens, the decrease was similar between cases and controls.

The distribution of test results for thyroid function among cases and controls at baseline and before delivery was examined in relation to the gestational age specific reference ranges for pregnant women. This was done to determine whether subgroups of the women who developed pre-eclampsia might have concentrations of thyroid hormones indicating the potential for clinically significant thyroid malfunction. Only the distributions of thyroid stimulating hormone concentration were significantly different between cases and controls. At baseline the proportion of women with thyroid stimulating hormone concentrations above the reference range did not differ between women who would later develop pre-eclampsia and controls 
Table 2|Results of thyroid function test in baseline and predelivery specimens and predelivery to baseline ratio in cases and controls. Values are geometric means (standard deviations) unless stated otherwise

\begin{tabular}{|c|c|c|c|c|c|c|c|c|c|}
\hline \multirow[b]{2}{*}{ Variables } & \multicolumn{3}{|c|}{ Baseline } & \multicolumn{3}{|c|}{ Predelivery } & \multicolumn{3}{|c|}{ Predelivery to baseline ratio* } \\
\hline & Controls & Cases & P value & Controls & Cases & P value & Controls & Cases & $\begin{array}{l}\text { Case ratio to control } \\
\text { ratio }(95 \% \mathrm{Cl})\end{array}$ \\
\hline No of women & 140 & 140 & - & 140 & 140 & & 141 & 139 & - \\
\hline Gestational age (days) $\ddagger$ & $112(20)$ & $111(20)$ & 0.92 & $260(18)$ & $265(22)$ & 0.04 & - & - & - \\
\hline TSH (mIU/l) & $1.22(0.27)$ & $0.99(0.22)$ & 0.14 & $2.44(0.67)$ & $3.15(0.83)$ & 0.007 & $1.48(0.11)$ & $2.42(0.24)$ & 1.64 (1.29 to 2.08$)$ \\
\hline Free T4 (pmol/l) & $13.87(0.41)$ & $13.81(0.41)$ & 0.81 & $12.30(0.82)$ & $12.10(0.77)$ & 0.45 & $0.86(0.01)$ & $0.85(0.01)$ & 0.99 (0.95 to 1.03$)$ \\
\hline Free T3 (pmol/l) & $4.94(0.14)$ & $5.09(0.15)$ & 0.11 & $3.98(0.23)$ & $3.95(0.22)$ & 0.70 & $0.89(0.01)$ & $0.85(0.01)$ & $0.96(0.92$ to 0.99$)$ \\
\hline hCG (IU/l) & 39051 (2539) & $43637(2837)$ & 0.23 & $16132(1290)$ & $21116(1688)$ & 0.02 & $0.42(0.04)$ & $0.48(0.04)$ & $1.15(0.91$ to 1.46$)$ \\
\hline
\end{tabular}

TSH=thyroid stimulating hormone; T4=thyroxine; T3=trriodothyronine; hCG=human chorionic gonadotrophin.

*Concentration units do not apply to ratios.

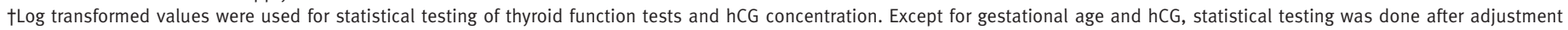

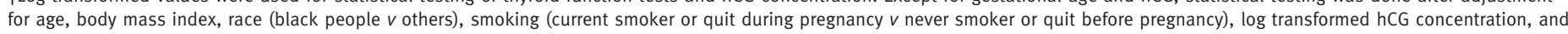
presence or absence of thyroid peroxidase antibodies above reference range.

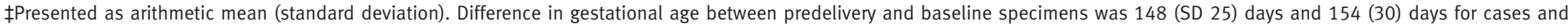
controls, respectively $(P=0.08)$. Two missing case specimens (one baseline, one predelivery) and their matched controls were excluded.

( $2 \%$ and $3 \%$, respectively, $\mathrm{P}=0.28$ after adjustment for age, body mass index, race, smoking, logarithmically transformed human chorionic gonadotrophin concentration, and the presence or absence of thyroid peroxidise antibodies). However, before delivery the proportion with thyroid stimulating hormone concentrations above the reference range was greater in the women with pre-eclampsia than in the controls: $24 \%$ of cases and 14\% of controls (arithmetic means 4.47 $\mathrm{mIU} / 1$ and $4.26 \mathrm{mIU} / \mathrm{l}$ ) had thyroid stimulating hormone concentrations above the reference range (adjusted $\mathrm{P}=0.03$ ). In predelivery specimens, but not at baseline, women with pre-eclampsia were significantly more likely than controls to have concentrations of thyroid stimulating hormone above the reference range (adjusted odds ratio 2.2, 95\% confidence interval 1.1 to 4.4$)$.

Of the 140 women with subsequent pre-eclampsia and 140 controls tested for thyroid peroxidase antibodies at baseline, six (4\%) in each group had positive results - that is, had levels above the reference range. In predelivery specimens, seven $(5 \%)$ cases and four $(3 \%)$ controls had positive results. The distributions of women with thyroid peroxidase antibodies above the upper limit of the reference range did not differ significantly between the women who developed preeclampsia and the normotensive controls.

At baseline none of the women had clinical hypothyroidism as defined by thyroid stimulating hormone concentration above the reference range and free thyroxine below. However, in predelivery specimens two women had developed clinical hypothyroidism (thyroid stimulating hormone 5.46 and $7.33 \mathrm{mIU} / 1$ and free thyroxine 8.0 and $8.2 \mathrm{pmol} / \mathrm{l}$ ), both after the onset of pre-eclampsia.

\section{Differences in thyroid stimulating hormone concentrations across quarters of soluble fms-like tyrosine kinase 1 concentrations}

To investigate mechanisms by which pre-eclampsia may be associated with the development of hypothyroidism, the differences between predelivery and baseline values of thyroid function tests were examined according to quarters of soluble fms-like tyrosine kinase 1 concentrations in the predelivery specimens. Cases and controls were analysed separately. Among controls the increase in thyroid stimulating hormone concentration between baseline and predelivery specimens was strongly associated ( $\mathrm{P}$ for trend $<0.001$ ) with increasing quarters of predelivery soluble fms-like tyrosine kinase 1 concentration: arithmetic means 0.01 (SD 1.14) mIU/l, 0.66 (1.04), 0.52 (0.75), and 0.92 (0.91), respectively (figure). The arithmetic mean of the difference in free thyroxine concentration between predelivery and baseline specimens was greater in the fourth quarter than in the first quarter $(-2.70 v-1.67$ $\mathrm{pmol} / \mathrm{l}, \mathrm{P}=0.03)$; but the test for trend was not significant. None of the other differences in thyroid function test results or the differences in human chorionic gonadotrophin concentration were associated with quarters of soluble fms-like tyrosine kinase 1 in predelivery specimens.

Among the cases with pre-eclampsia, the increase in thyroid stimulating hormone concentration between baseline and predelivery specimens was also strongly associated (P for trend 0.002) with increasing quarters of predelivery soluble fms-like tyrosine kinase: arithmetic means, respectively, 0.51 (SD 1.12) mIU/1, 0.94 (1.13) mIU/l, 1.41 (1.32) mIU/l, and 1.33 (1.29) mIU/l. Compared with controls, the increase in the preeclampsia group was consistently greater in each corresponding quarter. Differences between predelivery and baseline specimens for the other thyroid function tests and for human chorionic gonadotrophin were not associated with quarters of soluble fms-like tyrosine kinase 1 concentration in predelivery specimens.

\section{Nord-Trondelag Health Study}

Participants and specimens

Between 1995 and 1997 all inhabitants 20 years and older in Nord-Trondelag county in Norway were invited to participate in the Nord-Trondelag Health Study (HUNT-2). ${ }^{22}$ Nord-Trondelag county is characterised by a stable and ethnically homogeneous 

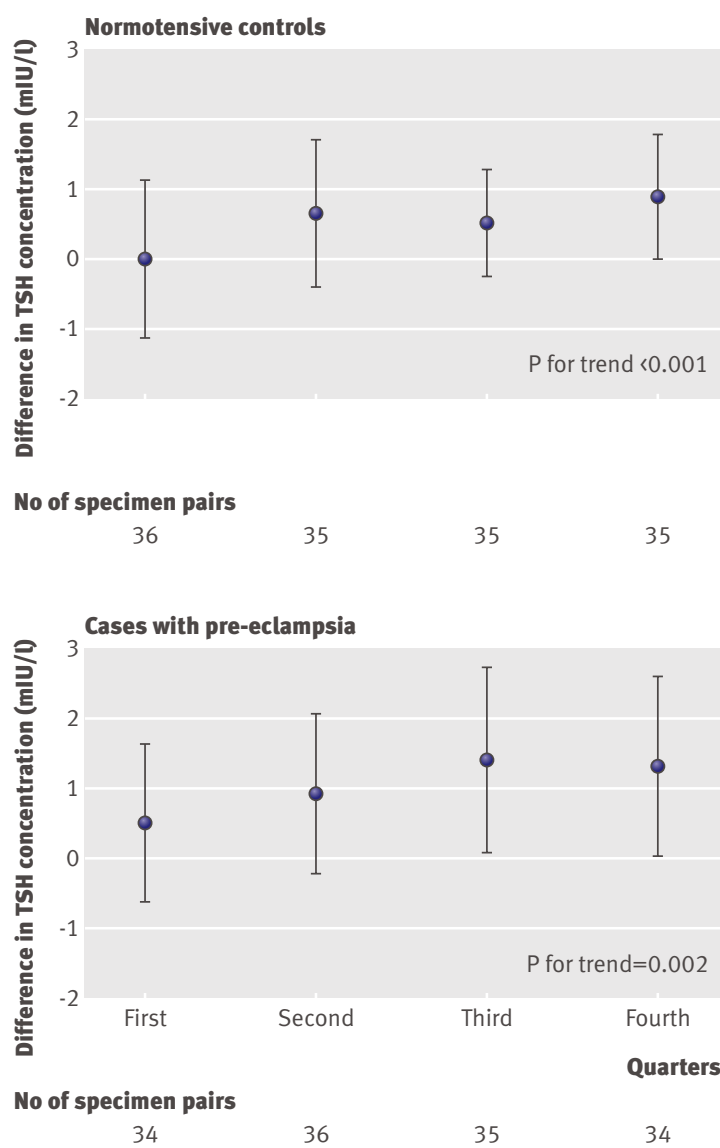

Comparisons of differences in thyroid stimulating hormone (TSH) concentration between predelivery and baseline specimens across quarters of predelivery soluble fms-like tyrosine kinase 1 concentrations in normotensive controls and cases with preeclampsia. |Soluble fms-like tyrosine kinase 1 concentrations in normotensive controls were $1710-5620 \mathrm{pg} / \mathrm{ml}$ in the first quarter, $5620-7554 \mathrm{pg} / \mathrm{ml}$ in the second quarter, $7554-$ $10789 \mathrm{pg} / \mathrm{ml}$ in the third quarter, and $10789-34907 \mathrm{pg} / \mathrm{ml}$ in the fourth quarter. Differences in the arithmetic mean thyroid stimulating hormone concentrations $(\mathrm{mIU} / \mathrm{l})$ for the first to fourth quarters were, respectively, $0.01,0.66(P=0.006), 0.52$ $(P=0.03)$, and 0.92 ( $P<0.001)$, with $P$ values indicated for comparisons with the first quarter. Soluble fms-like tyrosine kinase 1 concentrations in the cases with pre-eclampsia were $6620-13772 \mathrm{pg} / \mathrm{ml}$ in the first quarter, $13772-21282 \mathrm{pg} / \mathrm{ml}$ in the second quarter, $21282-30740 \mathrm{pg} / \mathrm{ml}$ in the third quarter, and $30740-82739 \mathrm{pg} / \mathrm{ml}$ in the fourth quarter. Differences in the arithmetic mean thyroid stimulating hormone concentrations ( $\mathrm{mlU} / \mathrm{l})$ for the first to fourth quarters were, respectively, $0.51,0.94,1.41(\mathrm{P}=0.003)$, and 1.33 $(P=0.006)$, with significant $P$ values indicated for comparisons with the first quarter

population, which is generally considered to have adequate iodine intake. A total of 92936 adults were eligible for the study, and $66140(71.2 \%)$ participated. The study has been described in detail elsewhere. ${ }^{2223} \mathrm{~A}$ non-fasting venous blood sample was requested from each participant. Thyroid stimulating hormone concentrations were determined in samples of the study population and included all women older than 40 years and a $5 \%$ random sample of women aged 20-40.
Linkage to medical birth registry of Norway

The unique 11 digit identification number of every Norwegian citizen enabled linkage of parous women to information in the medical birth registry of Norway. This nationwide registry has recorded data on all births in Norway since 1967. We therefore restricted the analysis to women who had had their first birth registered during the period from 1967 until participation in the Nord-Trondelag Health Study. Among women with a measurement for thyroid stimulating hormone concentration, 7933 had had their first birth in 1967 or later. We excluded women with known thyroid disease $(\mathrm{n}=695)$, twin or triplet first pregnancies $(\mathrm{n}=74)$, pregnancy at the time thyroid stimulating hormone concentration was determined $(\mathrm{n}=33)$, and women without information on smoking habits $(\mathrm{n}=10)$, leaving 7121 women for analysis. Thyroid peroxidase antibodies were also measured in women with a thyroid stimulating hormone concentration greater than $4 \mathrm{mIU} / \mathrm{l}$.

Criteria for pre-eclampsia used by the reporting midwives and obstetricians have been in accordance with the 1972 recommendations of the American College of Obstetrics and Gynecologists. ${ }^{24}$ These criteria include increased blood pressure after 20 weeks' gestation $(\geq 140 / 90 \mathrm{~mm} \mathrm{Hg}$, or an increase in systolic blood pressure of $\geq 30 \mathrm{~mm} \mathrm{Hg}$ or in diastolic blood pressure of $\geq 15 \mathrm{~mm} \mathrm{Hg}$ ), from measurements made before 20 weeks' gestation, and proteinuria $(\geq 0.3 \mathrm{~g}$ in a 24 hour urine specimen or a urine dipstick result of $\geq+$ ).

\section{Procedures}

Serum concentrations of thyroid stimulating hormone were measured at the Hormone Laboratory, Aker University Hospital, Oslo, using DELFIA hTSH Ultra (Wallac Oy, Turku, Finland). The coefficient of variation was less than $5 \%$. The clinical reference range for thyroid stimulating hormone in this population was defined as $0.50-3.50 \mathrm{mIU} / 1 .{ }^{25}$ Thyroid peroxidase antibodies were also measured in people with concentrations of thyroid stimulating hormone greater than 4 mIU/l (BRAHMS Diagnostica, Berlin, Germany). Those with levels greater than $200 \mathrm{IU} / \mathrm{ml}$ were considered to have tested positive for thyroid peroxidase antibodies.

\section{Statistical analysis}

We used multiple logistic regression analysis to determine odds ratios and 95\% confidence intervals. All analyses were adjusted for age and smoking status (current, former, or never).

\section{Results}

Analyses were carried out among 7121 women with thyroid stimulating hormone measurements who had delivered their first child during or after 1967, when the medical birth registry of Norway was established (table 3). Among women who had experienced preeclampsia in their first pregnancy the probability of having serum thyroid stimulating hormone concentrations greater than the clinical reference range $>3.5$ $\mathrm{mIU} / \mathrm{l})$ was higher than for women who did not 
Table $3 \mid$ Odds ratios for thyroid stimulating hormone (TSH) concentrations above the reference range ( $>3.5 \mathrm{mIU} / \mathrm{l})$, and for high TSH concentrations ( $\geq 4.0$ $\mathrm{mIU} / \mathrm{l})$ with positive $(>200 \mathrm{IU} / \mathrm{ml})$ or negative $(\leq 200 \mathrm{IU} / \mathrm{ml})$ results for thyroid peroxidase (TPO) antibody status, according to pre-eclampsia status in previous singleton pregnancies

\begin{tabular}{|c|c|c|c|c|c|c|}
\hline \multirow[b]{2}{*}{ Variables } & \multicolumn{2}{|c|}{ TSH concentration $>3.5 \mathrm{mIU} / \mathrm{l}$} & \multicolumn{2}{|c|}{$\begin{array}{l}\text { TSH concentration }>4.0 \mathrm{mlU} / \mathrm{l} \text {, } \\
\text { TPO antibodies }>200 \mathrm{IU} / \mathrm{ml}\end{array}$} & \multicolumn{2}{|c|}{$\begin{array}{l}\text { TSH concentration }>4.0 \mathrm{mIU} / \mathrm{l} \text {, } \\
\text { TPO antibodies } \leq 200 \mathrm{IU} / \mathrm{ml}\end{array}$} \\
\hline & Yes/No & Odds ratio $(95 \% \mathrm{Cl})$ & Yes/No & Odds ratio $(95 \% \mathrm{Cl})$ & Yes/No & Odds ratio $(95 \% \mathrm{Cl})$ \\
\hline \multicolumn{7}{|l|}{ Women with $\geq 1$ pregnancies: } \\
\hline No pre-eclampsia in first pregnancy & $407 / 6444$ & 1.0 (Reference) & $188 / 6651$ & 1.0 (Reference) & $89 / 6750$ & 1.0 (Reference) \\
\hline Pre-eclampsia in first pregnancy & $29 / 241$ & 1.7 (1.1 to 2.5$)$ & $14 / 255$ & $1.8(1.0$ to 3.1$)$ & $10 / 259$ & $2.6(1.3$ to 5.0$)$ \\
\hline \multicolumn{7}{|l|}{ Women with $\geq 2$ pregnancies: } \\
\hline No pre-eclampsia in first or second pregnancy & $350 / 5532$ & 1.0 (Reference) & $167 / 5704$ & 1.0 (Reference) & $72 / 5799$ & 1.0 (Reference) \\
\hline Pre-eclampsia in first or second pregnancy & $24 / 270$ & $1.3(0.9$ to 2.1$)$ & $12 / 281$ & $1.4(0.8$ to 2.5$)$ & $8 / 285$ & $2.2(1.0$ to 4.6$)$ \\
\hline Pre-eclampsia in first and second pregnancy & $4 / 21$ & 2.5 (0.9 to 7.5$)$ & $2 / 23$ & $2.6(0.6$ to 11.3$)$ & $2 / 23$ & 5.8 (1.3 to 25.5$)$ \\
\hline
\end{tabular}

Odds ratios are adjusted for age and smoking status at Nord-Trondelag Health Study examination.

develop pre-eclampsia in their first pregnancy (adjusted odds ratio 1.7, 95\% confidence interval 1.1 to 2.5). The mean number of years elapsed from delivery of the first pregnancy to the date of the thyroid stimulating hormone measurement was 20.4 among the women with pre-eclampsia in that pregnancy and 21.8 years among the women without pre-eclampsia in that pregnancy.

Thyroid peroxidase antibodies were also measured in women with thyroid stimulating hormone concentrations greater than $4.0 \mathrm{mIU} / \mathrm{l}$. The association of preeclampsia with subsequent hypothyroid function (thyroid stimulating hormone $>4.0 \mathrm{mIU} / \mathrm{l}$ ) was slightly stronger in the absence of thyroid peroxidase antibodies (adjusted odds ratio 2.6, 95\% confidence interval 1.3 to 5.0 ) than for hypothyroid function with thyroid peroxidase antibodies (1.8, 1.0 to 3.1 ; table 3$)$. Among women who had experienced pre-eclampsia in two pregnancies, the association with hypothyroid function in the absence of thyroid peroxidase antibodies was particularly strong $(5.8,1.3$ to 25.5$)$.

\section{DISCUSSION}

This study found that pre-eclampsia among nulliparous women is associated with a greater subsequent risk of subclinical hypothyroidism in pregnancy and that women with a history of pre-eclampsia are at greater risk of hypothyroid function many years after pre-eclampsia.

The findings during pregnancy are consistent with the results of previous studies ${ }^{26-28}$ and indicate that the increases in thyroid stimulating hormone concentration during pre-eclampsia are not related to changes in circulating human chorionic gonadotrophin concentrations, a protein that stimulates the thyroid gland and suppresses thyroid stimulating hormone. ${ }^{19}$ In women with pre-eclampsia, but also in normotensive controls, the extent of increase in thyroid stimulating hormone during pregnancy was directly related to the magnitude of circulating soluble fms-like tyrosine kinase 1 concentrations before delivery. This increase was substantially greater in the pre-eclampsia group, consistent with the suggestion that the effect of preeclampsia on thyroid function may be mediated by soluble fms-like tyrosine kinase 1 .
Norwegian women who had experienced preeclampsia in their first pregnancy were more likely than other women to have concentrations of thyroid stimulating hormone above the clinical reference range many years after the pregnancy. The association was stronger if the high concentration of thyroid stimulating hormone was combined with absence of thyroid peroxidase antibodies, and particularly strong if preeclampsia had occurred in two pregnancies. This suggests that the hypothyroid function associated with increased circulating concentrations of thyroid stimulating hormone in pre-eclampsia may occur independent of the autoimmune mechanisms that are generally accepted as the most likely cause of subclinical and overt hypothyroidism in iodine replete women. ${ }^{2930}$

The clinical syndrome of pre-eclampsia has been hypothesised to result from excessive release of antiangiogenic proteins - most notably soluble fms-like tyrosine kinase 1 -from the placenta into maternal blood, resulting in an antiangiogenic state with low levels of free placental growth factor and free vascular endothelial growth factor. ${ }^{831}$ Administration of vascular endothelial growth factor inhibitors such as soluble fms-like tyrosine kinase 1 to rodents induces hypertension, proteinuria, and glomerular endotheliosis, the hallmarks of pre-eclampsia. The particular sensitivity of glomerular capillaries to reduced levels of vascular endothelial growth factor may be attributed to their fenestrated endothelium, which requires the constitutive expression of vascular endothelial growth factor by renal podocytes for health and function. ${ }^{32}$ Thyroid capillaries also have a fenestrated endothelium. ${ }^{15}$ In mice, two weeks' exposure to exogenous soluble fmslike tyrosine kinase 1 or to other vascular endothelial growth factor inhibitors resulted in a reduction of thyroid tissue capillary density by two thirds and increased thyroid stimulating hormone concentration. Stopping soluble fms-like tyrosine kinase 1 from being administered led to a nearly complete recovery after three weeks. ${ }^{15}$ This seems to be analogous to the recovery from hypertension and proteinuria in experimental animals after stopping inhibition of vascular endothelial growth factor and in women with pre-eclampsia after delivery of the placenta. ${ }^{710}$ Together with reports of hypothyroidism in patients with cancer treated with 


\section{WHAT IS ALREADY KNOWN ON THIS TOPIC}

Limited data suggest that pre-eclampsia may be associated with hypothyroid function during pregnancy

Women with a history of pre-eclampsia are at increased risk of future cardiovascular and renal disease

\section{WHAT THIS STUDY ADDS}

Hypothyroid function during pre-eclampsia may result from the antiangiogenic state

Women with a history of pre-eclampsia may be at increased risk of future hypothyroid function

vascular endothelial growth factor receptor inhibitors, ${ }^{11-15}$ the evidence suggests that high levels of exposure to soluble fms-like tyrosine kinase 1 as in pre-eclampsia may be associated with increased risk for reduced thyroid function during and after pregnancy.

As women with subclinical thyroid dysfunction are at greater risk of progression to overt hypothyroidism, ${ }^{33}$ our findings could have important implications for the subsequent care of women with pre-eclampsia. Not only should they be followed closely for the development of cardiovascular and renal disease and the amelioration of predisposing risk factors, but consideration should also be given to monitoring for the development of reduced thyroid function and clinically important hypothyroidism. Although the absolute risk for reduced thyroid function and the future development of hypothyroidism in these women seems to be low, the availability of low cost thyroxine replacement therapy makes early treatment of hypothyroidism an attractive option that may substantially reduce the associated morbidity and improve quality of life. Moreover, untreated hypothyroidism may be a modifiable risk factor for cardiovascular disease; and its treatment might prevent early cardiovascular disease in women with a history of pre-eclampsia. ${ }^{23}$

Our study raises several questions: Is there a contribution of hypothyroid function to the excess of cardiovascular disease in women with a history of preeclampsia? Does subclinical hypothyroidism in women with pre-eclampsia have adverse effects on the long term health of the children ${ }^{34}{ }^{35}$ Does the risk of subclinical and overt hypothyroidism in women with pre-eclampsia increase with time elapsed after pregnancy? Are women who have had multiple normal pregnancies or pregnancies with multiple fetuses at greater risk of hypothyroidism because of the increased levels of soluble fms-like tyrosine kinase 1 in pregnancies with multiple fetuses and in normal pregnancies at term? The latter possibility is supported by our finding that soluble fms-like tyrosine kinase 1 concentration is positively associated with thyroid stimulating hormone concentration in normotensive pregnancies as well.

In summary, increased circulating concentrations of soluble fms-like tyrosine kinase 1 , most notably after onset of pre-eclampsia, were associated with subtle abnormalities of the thyroid during pregnancy. These in turn may predispose to the development of reduced thyroid function and possibly overt hypothyroidism in later life.

We thank the CPEP (Calcium for Pre-eclampsia Prevention) Study Group who assembled the database and specimen repository used here: I C Hauth, R Goldenberg, and B S Stofan (University of Alabama at Birmingham), L B Curet, G M Joffe, and V Dorato (University of New Mexico at Albuquerque), B M Sibai, SA Friedman, B M Mercer, and T Carr (University of Tennessee at Memphis), P M Catalano, A S Petrulis, and L Barabach (Case Western Reserve University at MetroHealth Medical Center, Cleveland), C Morris, S-L Jacobson, and K McCracken (Oregon Health Sciences University, Portland), J R Esterlitz, M G Ewell, and D M Brown (EMMES, Rockville), R J Levine, R DerSimonian, I D Clemens, M A Klebanoff, E G Raymond, J G Rigau-Perez, and H Shifrin (the Eunice Kennedy Shriver National Institute of Child Health), and J A Cutler and D E Bild (the National Heart, Lung, and Blood Institute); M Lindheimer, C Begg, T Chalmers, M Druzin, R Sokol (data safety and monitoring board); the participants; Frank Epstein, Nisha Parikh, Alexander Holston, Evelyn Wang, Hannah Elson, and Maren May for helpful discussions; Patricia Moyer for assistance with figures; and the Nord-Trondelag Health Study for permission to use the combined data with the medical birth registry of Norway.

Contributors: SAK and RJL had the original idea for the study. GLH carried out the thyroid function tests and measured the human chorionic gonadotrophin in the specimens from the Calcium for Pre-eclampsia Prevention trial. RJL, CQ, KFY, and SAK analysed data from the Calcium for Pre-eclampsia Prevention trial, and LJV, PRR, AlH, and BOA analysed the data from the Nord-Trondelag Health Study. RJL, SAK, and LJV wrote the paper assisted by GLH, CQ, PRR, KFY, ANH, BOA, and AlH, who also helped interpret the results. RJL has access to the original data from the Calcium for Pre-eclampsia Prevention trial and is guarantor of the Calcium for Pre-eclampsia Prevention trial results. LJV has access to the original data from the Nord-Trondelag Health Study and is guarantor of the NordTrondelag Health Study results.

Funding: This study was supported by the Eunice Kennedy Shriver National Institute of Child Health and Human Development (contract N01HD-5-3398) and by salary support from the intramural research programme of the National Institutes of Health. The Calcium for Preeclampsia Prevention trial was supported by the National Institute of Child Health and Human Development (contracts N01-HD-1-3121-3126, N01-HD-3154, and N01-HD-5-3246), with cofunding from the National Heart, Lung, and Blood Institute. SAK is supported by a clinical scientist award by the Burroughs Wellcome Fund and is an investigator of the Howard Hughes Medical Institute and an established investigator of the American Heart Association. The Nord-Trondelag Health Study is a collaborative effort of the Norwegian University of Science and Technology, the Norwegian Institute of Public Health, and the NordTrondelag County Council. This substudy of the Nord-Trondelag Health Study was supported by the Norwegian University of Science and Technology and by the Central Norway Regional Health Authority. Competing interests: SAK has served as a consultant to Abbott, Beckman Coulter, Roche, and Johnson \& Johnson and has been named coinventor on multiple provisional patents filed by Beth Israel Deaconess Medical Center for the use of angiogenesis related proteins for the diagnosis and treatment of pre-eclampsia. These patents have been non-exclusively licensed to several companies.

Ethical approval: Because the study used specimens that had been collected as part of the Calcium for Pre-eclampsia trial and could not be linked to identifiable women, the Office of Human Subjects Research of the National Institutes of Health granted an exemption from the requirement for review and approval by the institutional review board. Use of the Nord-Trondelag Health Study and Norwegian birth registry was approved by the Norwegian regional committee for medical research ethics and by the Norwegian Data Inspectorate.

1 Redman CW, Sargent IL. Latest advances in understanding preeclampsia. Science 2005;308:1592-4.

2 Sibai B, Dekker G, Kupferminc M. Pre-eclampsia. Lancet 2005;365:785-99.

3 Hubel CA, Snaedal S, Ness RB, Weissfeld LA, Geirsson RT, Roberts JM, et al. Dyslipoproteinaemia in postmenopausal women with a history of eclampsia. Br J Obstet Gynaecol 2000;107:776-84 
4 Sibai BM, el-Nazer A, Gonzalez-Ruiz A. Severe preeclampsiaeclampsia in young primigravid women: subsequent pregnancy outcome and remote prognosis. Am J Obstet Gynecol 1986;155:1011-6.

5 Irgens HU, Reisaeter L, Irgens LM, Lie RT. Long term mortality of mothers and fathers after pre-eclampsia: population based cohort study. BMJ 2001;323:1213-7.

6 Vikse BE, Irgens LM, Leivestad T, Skjaerven R, Iversen BM. Preeclampsia and the risk of end-stage renal disease. N Engl J Med 2008;359:800-9.

7 Maynard SE, Min JY, Merchan J, Lim KH, Li J, Mondal S, et al. Excess placental soluble fms-like tyrosine kinase 1 (sFlt1) may contribute to endothelial dysfunction, hypertension, and proteinuria in preeclampsia. J Clin Invest 2003;111:649-58.

8 Levine RJ, Maynard SE, Qian C, Lim KH, England LJ, Yu KF, et al. Circulating angiogenic factors and the risk of preeclampsia. $N$ Engl J Med 2004;350:672-83.

9 Chaiworapongsa T, Romero R, Espinoza J, Bujold E, Mee Kim Y, Goncalves LF, et al. Evidence supporting a role for blockade of the vascular endothelial growth factor system in the pathophysiology of preeclampsia. Young investigator award. Am J Obstet Gynecol 2004;190:1541-7.

10 Eremina V, Jefferson JA, Kowalewska J, Hochster H, Haas M, Weisstuch J, et al. VEGF inhibition and renal thrombotic microangiopathy. N Engl J Med 2008;358:1129-36.

11 Kamba T, McDonald DM. Mechanisms of adverse effects of anti-VEGF therapy for cancer. Br J Cancer 2007;96:1788-95.

12 Desai J, Yassa L, Marqusee E, George S, Frates MC, Chen MH, et al. Hypothyroidism after sunitinib treatment for patients with gastrointestinal stromal tumors. Ann Intern Med 2006;145:660-4.

13 Wolter P, Stefan C, Decallonne B, Dumez H, Bex M, Carmeliet P, et al. The clinical implications of sunitinib-induced hypothyroidism: a prospective evaluation. Br J Cancer 2008;99:448-54.

14 Feldman DR, Baum MS, Ginsberg MS, Hassoun H, Flombaum CD, Velasco S, et al. Phase I trial of bevacizumab plus escalated doses of sunitinib in patients with metastatic renal cell carcinoma. J Clin Oncol 2009;27:1432-9.

15 Kamba T, Tam BY, Hashizume H, Haskell A, Sennino B, Mancuso MR, et al. VEGF-dependent plasticity of fenestrated capillaries in the normal adult microvasculature. Am J Physiol Heart Circ Physiol 2006;290:H560-76.

16 Levine RJ, Esterlitz JR, Raymond EG, DerSimonian R, Hauth JC, Curet LB, et al. Trial of calcium for preeclampsia prevention (CPEP): rationale, design, and methods. Control Clin Trials 1996;17:442-69.

17 Levine RJ, Hauth JC, Curet LB, Sibai BM, Catalano PM, Morris CD, et al. Trial of calcium to prevent preeclampsia. N Engl J Med 1997;337:69-76.

18 Holston AM, Qian C, Karumanchi SA, Yu KF, Levine RJ. Circulating angiogenic factors in gestational proteinuria without hypertension. Am J Obstet Gynecol 2009;200:392 e1-10.

19 Burrow GN, Fisher DA, Larsen PR. Maternal and fetal thyroid function. N Engl J Med 1994;331:1072-8.
20 Roche Diagnostics. Reference intervals for children and adults. In: Elecsys thyroid tests. Mannheim, Germany: Roche Diagnostics, 2004:5.

21 Zhang J, Bowes WA Jr. Birth-weight-for-gestational-age patterns by race, sex, and parity in the United States population. Obstet Gynecol 1995;86:200-8

22 Holmen J, Midthjell K, Kruger O, Langhammer A, Holmen TL, Bratberg GH, et al. The Nord-Trondelag Health Study 1995-97 (HUNT 2): objectives, contents, methods and participation. Norsk Epidemol 2003;13:19-32.

23 Asvold BO, Bjoro T, Nilsen TI, Gunnell D, Vatten LI. Thyrotropin levels and risk of fatal coronary heart disease: the HUNT study. Arch Intern Med 2008;168:855-60.

24 National High Blood Pressure Education Program Working Group. Report on high blood pressure in pregnancy. Am J Obstet Gynecol 1990;163:1691-712.

25 Bjoro T, Holmen J, Kruger O, Midthjell K, Hunstad K, Schreiner T, et al. Prevalence of thyroid disease, thyroid dysfunction and thyroid peroxidase antibodies in a large, unselected population: the Health Study of Nord-Trondelag (HUNT). Eur J Endocrinol 2000;143:639-47.

26 Larijani B, Marsoosi V, Aghakhani S, Moradi A, Hashemipour S. Thyroid hormone alteration in pre-eclamptic women. Gynecol Endocrinol 2004;18:97-100.

27 Lao T, Chin RK, Swaminathan R, Lam YM. Maternal thyroid hormones and outcome of pre-eclamptic pregnancies. Br J Obstet Gynaecol 1990;97:71-4.

28 Cleary-Goldman J, Malone FD, Lambert-Messerlian G, Sullivan L, Canick J, Porter TF, et al. Maternal thyroid hypofunction and pregnancy outcome. Obstet Gynecol 2008;112:85-92.

29 Hollowell JG, Staehling NW, Flanders WD, Hannon WH, Gunter EW, Spencer CA, et al. Serum TSH, T(4), and thyroid antibodies in the United States population (1988 to 1994): National Health and Nutrition Examination Survey (NHANES III). J Clin Endocrinol Metab 2002;87:489-99.

30 Roberts CG, Ladenson PW. Hypothyroidism. Lancet 2004;363:793-803.

31 Maynard S, Epstein FH, Karumanchi SA. Preeclampsia and angiogenic imbalance. Annu Rev Med 2008;59:61-78.

32 Risau W. Development and differentiation of endothelium. Kidney Int 1998;67:S3-6.

33 Biondi B, Cooper DS. The clinical significance of subclinical thyroid dysfunction. Endocr Rev 2008;29:76-131.

34 Casey BM, Dashe JS, Wells CE, McIntire DD, Byrd W, Leveno KJ, et al. Subclinical hypothyroidism and pregnancy outcomes. Obstet Gynecol 2005;105:239-45.

35 Haddow JE, Palomaki GE, Allan WC, Williams JR, Knight G], Gagnon et al. Maternal thyroid deficiency during pregnancy and subsequent neuropsychological development of the child. $N$ Engl J Med 1999;341:549-55

Accepted: 29 June 2009 